\title{
ARTICLE
}

Received 2 Dec 2013 | Accepted 5 Feb 2014 | Published 25 Feb $2014 \quad$ DOl: 10.1038/ncomms4389

\section{Improving the stability and optical properties of germanane via one-step covalent methyl-termination}

Shishi Jiang ${ }^{1}$, Sheneve Butler ${ }^{1}$, Elisabeth Bianco', Oscar D. Restrepo ${ }^{2}$, Wolfgang Windl ${ }^{2}$ \& Joshua E. Goldberger ${ }^{1}$

Two-dimensional van der Waals materials have shown great promise for a variety of electronic, optoelectronic, sensing and energy conversion applications. Since almost every atom in these two-dimensional crystals is exposed to the surface, covalent surface termination could provide a powerful method for the controlled tuning of material properties. Here we demonstrate a facile, one-step metathesis approach that directly converts $\mathrm{CaGe}_{2}$ crystals into mm-sized crystals of methyl-terminated germanane $\left(\mathrm{GeCH}_{3}\right)$. Replacing $-\mathrm{H}$ termination in $\mathrm{GeH}$ with $-\mathrm{CH}_{3}$ increases the band gap by $\sim 0.1 \mathrm{eV}$ to $1.7 \mathrm{eV}$, and produces band edge fluorescence with a quantum yield of $\sim 0.2 \%$, with little dependence on layer thickness. Furthermore, the thermal stability of $\mathrm{GeCH}_{3}$ has been increased to $250^{\circ} \mathrm{C}$ compared with $75^{\circ} \mathrm{C}$ for $\mathrm{GeH}$. This one-step metathesis approach should be applicable for accessing new families of two-dimensional van der Waals lattices that feature precise organic terminations and with enhanced optoelectronic properties.

\footnotetext{
${ }^{1}$ Department of Chemistry and Biochemistry, The Ohio State University, Columbus, Ohio 43210, USA. ${ }^{2}$ Department of Materials Science and Engineering The Ohio State University, Columbus, Ohio 43210, USA. Correspondence and requests for materials should be addressed to J.E.G.

(email: goldberger.4@osu.edu).
} 
$\mathrm{T}$ here has been remarkable widespread interest in the exploration of the unique properties and applications of single and few-layer thick sheets of layered van der Waals materials such as graphene or the layered transition metal dichalcogenides ${ }^{1-7}$. This work has shown the significant role of the immediate environment on the properties and reactivity of these van der Waals layers ${ }^{8}$. This suggests, on the other hand, the intriguing possibility of manipulating the properties of singleatom thick materials by covalent termination with rationally designed substituents. In contrast to the negligible role the surface binding ligand plays in nanoscale materials, terminating the surface of single-atom thick materials with different ligands is predicted to allow for the broad tuning of properties including band gap, band alignment, thermal stability, carrier mobility and spin-dependent interactions $s^{9,10}$. For example, a quantum spin Hall state with a surface-tunable spin-orbit gap has been recently predicted for two-dimensional (2D) Sn graphene analogues that are terminated with halides but not with hydrogen ${ }^{11}$, opening the door for novel lateral heterostructures between topological and conventional states. Unfortunately, most of the 2D materials studied to date comprise neutral van der Waals layers that lack the possibility of covalent functionalization. Although the functionalization of graphene with organic components, hydrogen atoms or even halogens have been achieved, these modifications completely disrupt the excellent electronic mobility of the Fermi-Dirac state, to produce $3-5 \mathrm{eV}$ band gap semiconductors ${ }^{12-15}$.

Researchers have recently developed layered van der Waals systems in which each surface atom requires a covalent ligand to become coordinatively saturated, such as the metal carbide/ nitride MXenes, the amine coordinated II-VI chalcogenides, and the group IV graphene analogues such as silicane or germanane $e^{9,16-20}$. Because the valence/conduction bands of these materials are comprised of metal-metal or metal-anion bonding within the $2 \mathrm{D}$ plane $\mathrm{e}^{21}$, rather than the $\pi$-bands such as that in graphene, these systems offer the possibility to tune the entire electronic properties on the basis of the identity and electron withdrawing capability of the substituent, without completely disrupting the relevant electronic states. The creation of many of these surface-terminated layered materials often relies on topotactic methods to interconvert a precursor layered crystal structure directly into the van der Waals material, frequently through deintercalation in acidic aqueous conditions that can result in partial surface termination with unwanted oxide or hydroxide states. For example, the deintercalation of layered Zintl phases, such as $\mathrm{CaSi}_{2}$ in aqueous $\mathrm{HCl}$ produces the air and water reactive, partially hydroxide-terminated silicane $\mathrm{SiH}_{\mathrm{x}}(\mathrm{OH})_{1-\mathrm{x}}$ as evidenced by the existence of broad $\mathrm{Si}-\mathrm{O}$ stretches that occur between $1,000-1,200 \mathrm{~cm}^{-1}$ in the Fourier Transform Infrared (FTIR) spectrum ${ }^{19,20}$. This ambiguity in surface functionalization convolutes efforts to correlate the effects of surface functionalization on the optoelectronic properties of these single-atom thick semiconductors ${ }^{9,20,22-24}$. Furthermore, it is well established that H-terminated $\mathrm{Si}(111)$ and $\mathrm{Ge}(111)$ surfaces are extremely air reactive, whereas the $-\mathrm{CH}_{3}$ terminated surfaces can be resilient towards oxidation $^{25,26}$. To bypass the potential air-reactive intermediates and create new organic-terminated materials, new single-step synthetic methods that can directly convert a precursor crystalline solid-state crystal into a crystalline, exfoliatable, organicterminated van der Waals solid in nonaqueous solvents is required. Towards these ends, we report a one-step metathesis
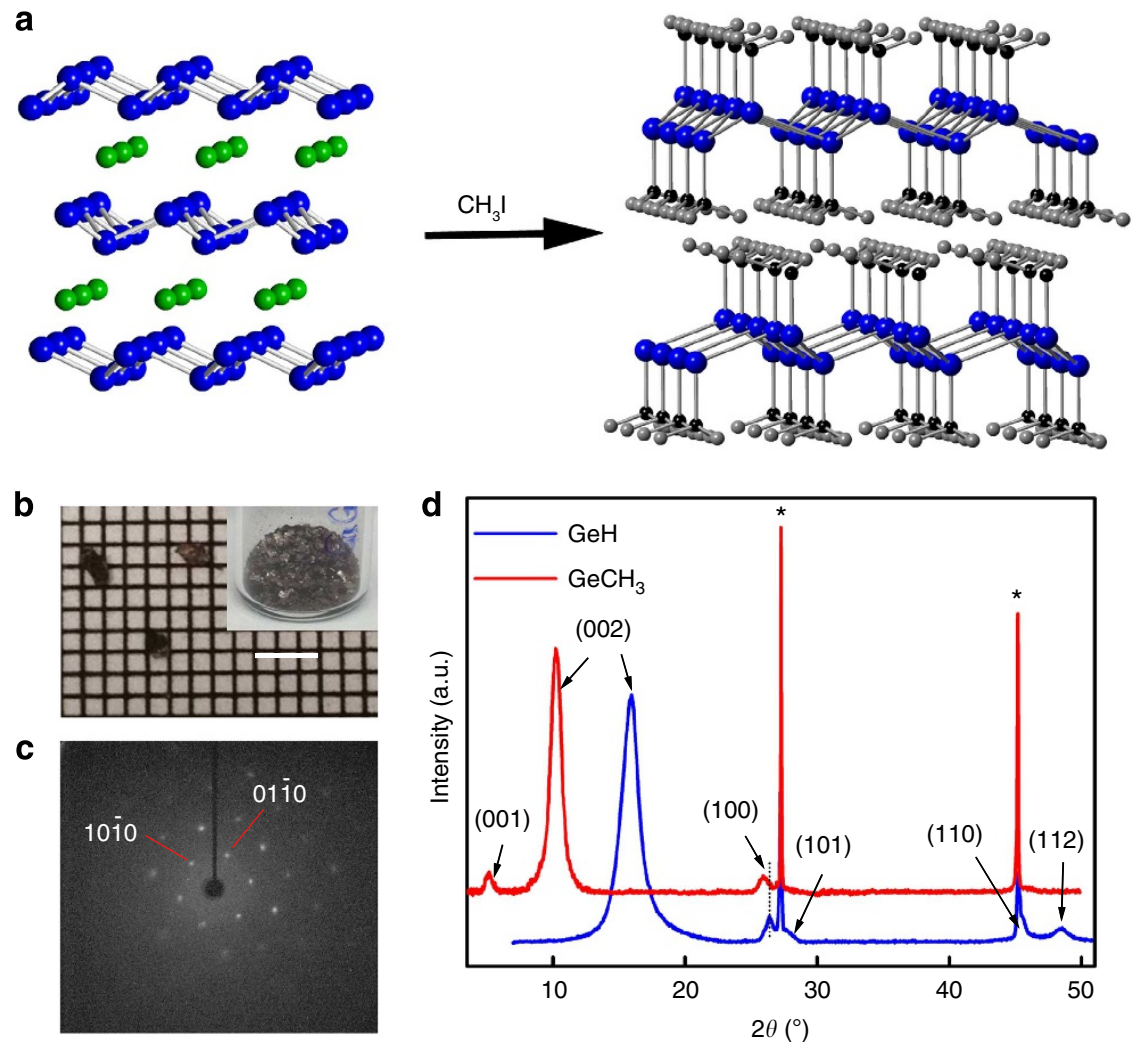

Figure 1 | The one-step topotactic transformation of $\mathbf{C a G e}_{\mathbf{2}}$ into $\mathbf{G e C H}_{\mathbf{3}}$ crystals. (a) Schematic illustration of conversion of $\mathrm{CaGe} 2$ (left) into $\mathrm{GeCH}_{3}$ (right). (b) Optical images of $\mathrm{GeCH}_{3}$ crystals with select crystals on graph paper with a $1 \mathrm{~mm}$ grid. Scale bar, $3 \mathrm{~mm}$. (c) Single-crystal XRD pattern of $\mathrm{GeCH}_{3}$ collected down the [001] zone axis. (d) Powder XRD patterns of $\mathrm{GeH}$ (blue) and $\mathrm{GeCH}_{3}$ (red). The starred peaks correspond to diffraction reflections of an internal $\mathrm{Ge}$ standard. The dotted line highlights the changes in the 100 reflections between $\mathrm{GeH}$ and $\mathrm{GeCH}$. XRD, X-ray diffraction. 
approach that directly converts $\mathrm{CaGe}_{2}$ into $\mathrm{GeCH}_{3}$, a methylterminated layered van der Waals solid.

\section{Results}

Structural characterization. The crystal structure of $\mathrm{CaGe}_{2}$ consists of hexagonal, puckered $\mathrm{sp}^{3}$ layers of $\mathrm{Ge}^{-}$atoms that are separated by $\mathrm{Ca}^{2+}$ ions (Fig. 1a). We hypothesized that the anionic $\mathrm{Ge}^{-}$on the surface and edges of the crystals could react topotactically in an $\mathrm{S}_{\mathrm{N}} 2$ or metathesis-like fashion with a small organic molecule, such as $\mathrm{CH}_{3} \mathrm{I}$, to form a $\mathrm{Ge}-\mathrm{CH}_{3}$ bond along with $\mathrm{CaI}_{2}$. The expansion of the lattice would allow the precursors to diffuse inward and the reaction would proceed to completion. However, in our initial experiments with pure $\mathrm{CH}_{3} \mathrm{I}$, only the surface layers of $\mathrm{CaGe}_{2}$ had reacted, likely due to the low solubility of $\mathrm{CaI}_{2}$. Instead, we developed a biphasic $\mathrm{CH}_{3} \mathrm{I} / \mathrm{H}_{2} \mathrm{O}$ solvent reaction in which $2-3 \mathrm{~mm}$ crystals of $\mathrm{CaGe}_{2}$ are fully immersed in $\mathrm{CH}_{3} \mathrm{I}$, while $\mathrm{CaI}_{2}$ is transferred into the $\mathrm{H}_{2} \mathrm{O}$ layer (Supplementary Fig. 1). After rinsing in concentrated $\mathrm{HCl}$ to remove any trace residual $\mathrm{CaI}_{2}$, and then isopropanol, the reaction yields crystals of $\mathrm{GeCH}_{3}$ that are $\sim 1 \mathrm{~mm}$ in diameter and $<100 \mu \mathrm{m}$ in thickness (Fig. 1b and Supplementary Fig. 2). Singlecrystal X-ray diffraction (XRD) analysis (Fig. 1c) shows that each one of these crystallites is a single crystal having a hexagonal spacing of $a=3.96 \AA$; however, the interlayer turbostratic disorder and curvature of these crystallites preclude determination of the $c$ axis spacing. Powder XRD analysis (Fig. 1d) of $\mathrm{GeCH}_{3}$ confirms that it can be fit to a $2 \mathrm{H}$ unit cell (two $\mathrm{GeCH}_{3}$ layers per hexagonal unit cell spacing) with $a=3.97 \AA$ and $c=17.26 \AA$ (8.63 $\AA$ per layer). This corresponds to a $0.09 \AA$ expansion in the a-direction and a $3.1 \AA$ increase per layer compared to $\mathrm{GeH}$, which has lattice parameters of $a=3.880 \AA$ and $c=11.04 \AA$ $(5.50 \AA \text { per layer })^{9}$. This $3.1 \AA$ increase is close to twice the difference between the $\mathrm{Ge}-\mathrm{C}$ bond length $(1.95 \AA)$ and the $\mathrm{Ge}-\mathrm{H}$ bond length $(1.52 \AA)$ plus twice the difference between the van der Waals radii of $-\mathrm{CH}_{3}(2.0 \AA)$ and $-\mathrm{H}(1.2 \AA)$, further indicating substitution of the $-\mathrm{H}$ substituent with a $-\mathrm{CH}_{3}$ substituent (Supplementary Fig. 3).

Transmission-mode Fourier Transform Infrared spectroscopy further confirms the $-\mathrm{CH}_{3}$ surface termination in $\mathrm{GeCH}_{3}$ (Fig. 2a) and that our sample is free of residual oxide. $\mathrm{In}_{\mathrm{GeCH}}$, the major Ge-H stretching frequency ${ }^{9,24,27-29}$ at $\sim 2,000 \mathrm{~cm}^{-1}$ is almost completely gone and replaced by a $\mathrm{Ge}-\mathrm{C}$ stretch ${ }^{29}$ that occurs at $573 \mathrm{~cm}^{-1}$. The other major modes that are observed in $\mathrm{GeCH}_{3}$ correspond to $-\mathrm{CH}_{3}$ stretching at 2,907 and 2,974 $\mathrm{cm}^{-1}$, $-\mathrm{CH}_{3}$ bending at 1,403 and $1,237 \mathrm{~cm}^{-1}$ and $-\mathrm{CH}_{3}$ rocking at $778 \mathrm{~cm}^{-1}$ (refs 29,30). The residual amount of $\mathrm{Ge}-\mathrm{H}$ stretching suggests that there exists a small percentage of $-\mathrm{H}$ termination, and elemental analysis suggests that $90 \% \pm 10 \%$ of the Germanium atoms are terminated with $-\mathrm{CH}_{3}$. This residual $-\mathrm{H}$ could result either from the minor solubility of $\mathrm{H}_{2} \mathrm{O}$ in $\mathrm{CH}_{3} \mathrm{I}$, or during the washing process. To further confirm the identity of each vibrational mode, we also created $\mathrm{Ge}^{13} \mathrm{CH}_{3}$ and $\mathrm{GeCD}_{3}$. In $\mathrm{Ge}^{13} \mathrm{CH}_{3}$, the $\mathrm{Ge}-\mathrm{C}$ stretch shifts down to $558 \mathrm{~cm}^{-1}$, and the other $-\mathrm{CH}_{3}$ vibrational modes slightly decrease by $1-10 \mathrm{~cm}^{-1}$ (Supplementary Table 1). There is a much more significant change in the vibrational energies of $\mathrm{GeCD}_{3}$, as the $-\mathrm{CD}_{3}$ stretching modes are shifted to 2,240 and $2,116 \mathrm{~cm}^{-1}$, the $-\mathrm{CD}_{3}$ bending mode decreases to 1,024 and $954 \mathrm{~cm}^{-1}$, the $-\mathrm{CD}_{3}$ rocking mode decreases to $584 \mathrm{~cm}^{-1}$, and the $\mathrm{Ge}-\mathrm{CD}_{3}$ stretch decreases to $530 \mathrm{~cm}^{-1}$ (Supplementary Table 1) ${ }^{29}$. As the $778 \mathrm{~cm}^{-1} \mathrm{CH}_{3}$ rocking mode can possibly mask the existence of any residual $\mathrm{Ge}-\mathrm{O}-\mathrm{Ge}$ or $\mathrm{Ge}-\mathrm{O}$ vibrational modes which normally occur from $800-1,000 \mathrm{~cm}^{-1}$ (refs 24,28), the shift of this rocking mode in $\mathrm{GeCD}_{3}$ allows elucidation of any residual $\mathrm{Ge}-\mathrm{O}-\mathrm{Ge}$ or $\mathrm{Ge}-\mathrm{O}$. The only vibrational modes observed in this
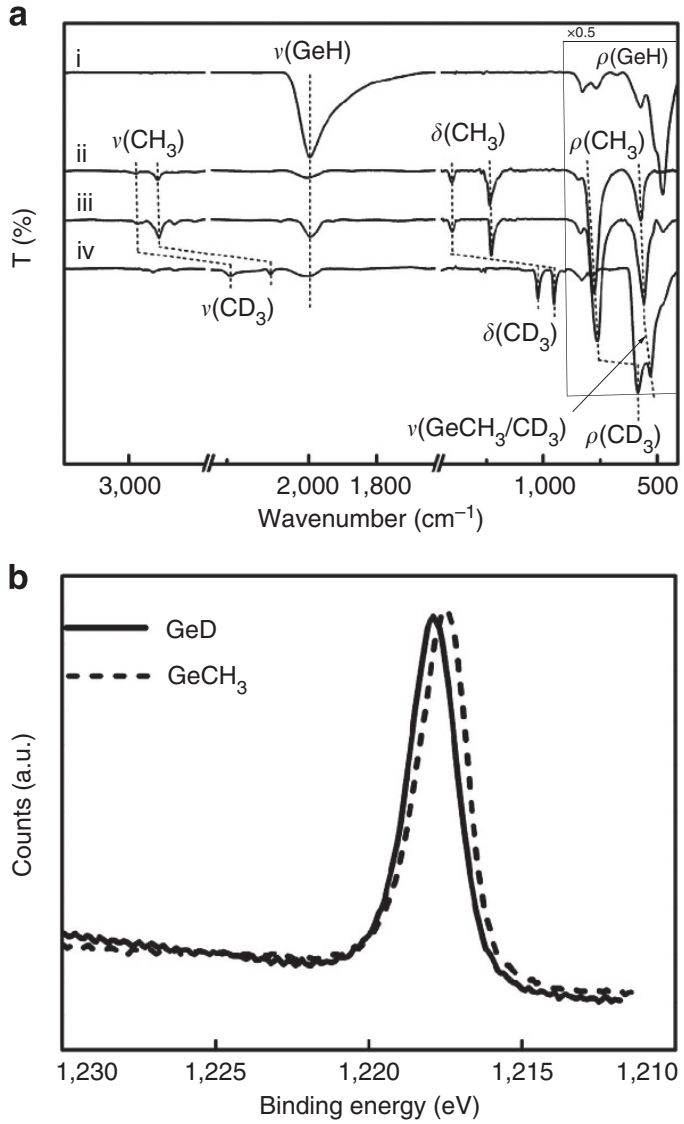

Figure 2 | The surface terminating chemistry of $\mathbf{G e C H}_{3}$. (a) FTIR spectra of $\mathrm{GeH}$ (i), $\mathrm{GeCH}_{3}$ (ii), $\mathrm{Ge}^{13} \mathrm{CH}_{3}$ (iii) and $\mathrm{GeCD}_{3}$ (iv). The intensity of the four spectra are all multiplied by 0.5 in the range of $400-900 \mathrm{~cm}^{-1}$. (b) XPS spectra of the $\mathrm{Ge} 2 \mathrm{p}_{3 / 2}$ peak for $\mathrm{GeH}$ (solid line) and $\mathrm{GeCH}_{3}$ (dashed line), indicating exclusively $\mathrm{Ge}^{+1}$ on the surface. XPS, X-ray photoelectron spectroscopy.

region for $\mathrm{GeCD}_{3}$ are the 770 and $830 \mathrm{~cm}^{-1}$ vibrations that correspond to bond-bending $\mathrm{Ge}-\mathrm{H}_{2}$ modes from nearest neighbour Ge atoms at the crystal edges $9,27,28$. X-ray Photoelectron Spectroscopy (XPS) measurements indicate a single Germanium +1 oxidation state (Fig. $2 \mathrm{~b}$ ), further suggesting that $\mathrm{GeO}_{2}$ and other surface oxides are not present. The $\mathrm{Ge} 2 \mathrm{p}_{3 / 2}$ peak occurs at $1,217.5 \mathrm{eV}$, which is slightly shifted compared with the observed $1,217.8 \mathrm{eV}$ peak of $\mathrm{GeH}$, but consistent with $\mathrm{CH}_{3}$-terminated $\mathrm{Ge}(111)$. This slight shift to lower XPS binding energy is consistent with previously observed XPS spectra of $-\mathrm{H}$ or $-\mathrm{CH}_{3}$ terminated silicon surfaces ${ }^{25}$.

Optical properties and band structure. The absorption and fluorescence measurements of $\mathrm{GeCH}_{3}$ are consistent with that of a direct band gap semiconductor. $\mathrm{GeCH}_{3}$ has strong photoluminescence (PL) emission centred at $1.7 \mathrm{eV}(730 \mathrm{~nm}$ (red)), which is close to the observed diffuse reflectance absorption (DRA) onset at $1.69 \mathrm{eV}$ (Fig. 3a). This corresponds to a $0.1 \mathrm{eV}$ increase in band edge compared with $\mathrm{GeH}(1.59 \mathrm{eV})$ (Fig. 3b). Band structure calculations for the measured structure using the hybrid HSE06 (refs 31,33) exchange-correlation functional, confirm this band gap, and predict that the two-layer unit cell has a direct band gap of $1.82 \mathrm{eV}$ (Fig. 3c). The red PL can be easily detected by eye under UV illumination in both solid-state samples and in suspension in isopropanol (Fig. 3d). The full-width at half maximum of the fluorescence emission is $\sim 250 \mathrm{meV}$. The absolute quantum yield of the solid flakes was 

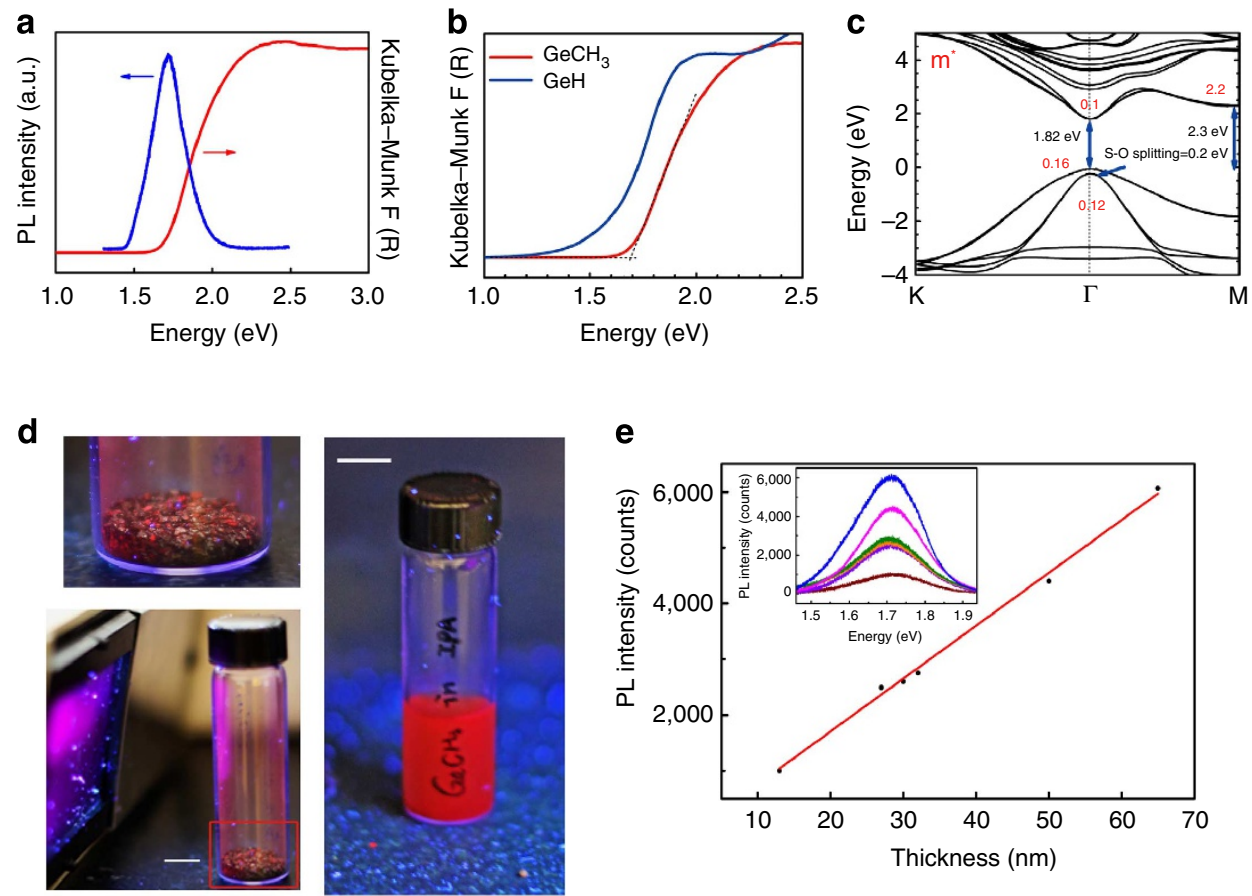

Figure 3 | The optical properties and band structure of $\mathbf{G e C H}_{3}$. (a) DRA (red) and PL (blue) of $\mathrm{GeCH}_{3}$. (b) DRA spectra of GeH (blue) and $\mathrm{GeCH}_{3}$ (red) plotted in Kubelka-Munk function versus photon energy. (c) Electronic band structure of a bilayer $\mathrm{GeCH}_{3}$ unit cell calculated using the hybrid HSEO6 theory including spin-orbit coupling with experimental lattice parameters ( $3.96 \AA$ ) predicting a $1.82 \mathrm{eV}$ direct band gap. The hole and electron effective masses for each extrema are indicated in red. (d) Images of $\mathrm{GeCH}_{3}$ photoluminescence of crystals (left) and in suspension in isopropanol (right), upon illumination with a handheld $365 \mathrm{~nm}$ light. Scale bars, $1 \mathrm{~cm}$. (e) PL intensity of exfoliated $\mathrm{GeCH}_{3}$ thin flakes having average thicknesses ranging from $13-65 \mathrm{~nm}$. Inset is the raw photoluminescence spectra of flakes with the thickness of $13 \mathrm{~nm}$ (wine), $27 \mathrm{~nm}$ (violet), $29 \mathrm{~nm}$ (orange), $31 \mathrm{~nm}$ (olive), $49 \mathrm{~nm}$ (magenta) and $65 \mathrm{~nm}$ (blue).
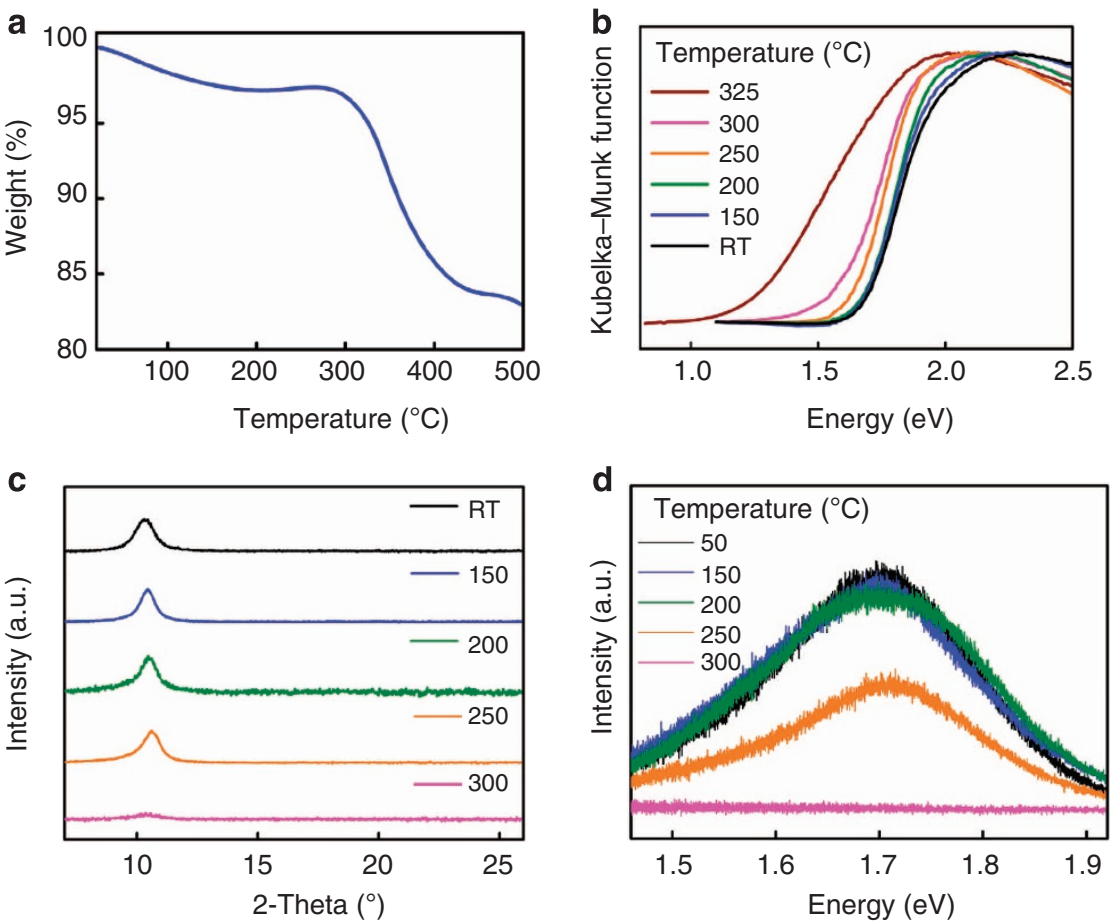

Figure 4 | The enhanced thermal stability of $\mathbf{G e C H}_{\mathbf{3}}$. (a) Thermogravimetric analysis of $\mathrm{GeCH}_{3}$. (b,c) DRA spectra (b) and powder XRD patterns (c) of bulk $\mathrm{GeCH}_{3}$ after annealing in $5 \% \mathrm{H}_{2} / \mathrm{Ar}$ for four hours at various temperatures. (d) Photoluminescence spectra of a single exfoliated $\mathrm{GeCH}_{3}$ flake using the same annealing procedure. 
measured to be $0.23 \%$. This represents a minimum bound of the quantum yield, due to the difficulty in correcting for selfabsorption in solid-state measurements. The full-width at half maximum and quantum yield values are close to those observed in exfoliated single-layer $\mathrm{MoS}_{2}$, which are $50-150 \mathrm{meV}$, and $0.4-0.5 \%{ }^{34,35}$, respectively. We hypothesize that even further improvements in the quantum yield could arise with better control over the degree of $-\mathrm{CH}_{3}$ versus $-\mathrm{H}$ functionalization. In $\mathrm{MoS}_{2}$ and many other metal dichalcogenides, a direct band gap is only observed when exfoliated down to single layers ${ }^{36}$, making the preparation of large-area single layers a necessary and challenging requirement before optoelectronic devices can be fabricated. In contrast, we observe that the photoluminescence emission intensity of exfoliated samples is linearly proportional to the number of layers from 13-65 layers (Fig. 3e and Supplementary Fig. 4). The band edge emission does not depend on layer thickness, at least with 13 layers and above, reflecting the relatively weak electronic coupling and orbital overlap of the conduction and valence bands in neighbouring layers. This is in contrast to the overlap of neighbouring $C 2 \mathrm{p}_{\mathrm{z}}$ orbitals in graphite, as well as the $S 3 p_{z}$ orbitals at the $\Gamma$ point in $\mathrm{MoS}_{2}$ (ref. 36). Finally, the intense PL contrasts with our observations on our previously reported crystals of $\mathrm{GeH}$, of which we have yet to observe any band edge $\mathrm{PL}^{9}$. Taken together, this data shows that the nature of the covalently modifiable surface ligand can tune the optoelectronic properties of these materials.

Thermal stability. We previously observed that $\mathrm{GeH}$ begins to amorphize upon annealing at $75^{\circ} \mathrm{C}$, is completely amorphous above $175^{\circ} \mathrm{C}$ and starts to dehydrogenate between $200^{\circ} \mathrm{C}$ and $250^{\circ} \mathrm{C}^{9}$. In contrast, $\mathrm{GeCH}_{3}$ has considerably enhanced thermal stability. According to thermogravimetric analysis, a transition occurs starting at $300^{\circ} \mathrm{C}$ (Fig. 4a) that corresponds to the expected mass loss for approximately $90 \% \mathrm{CH}_{3}$-termination, which is in excellent agreement with our elemental analysis. We have previously found that DRA is a much more sensitive probe of the degree of amorphization than any other technique, due to the reduced band gap of amorphous germanium 9 . There is virtually no shift in band edge emission up to $200^{\circ} \mathrm{C}$, whereas after annealing at $250{ }^{\circ} \mathrm{C}$ and $300^{\circ} \mathrm{C}$, the band edge red-shifts by 0.06 and $0.10 \mathrm{eV}$, respectively (Fig. $4 \mathrm{~b}$ ). The powder XRD pattern also shows negligible changes after annealing up to $250{ }^{\circ} \mathrm{C}$, but it is almost completely amorphous after annealing at $300^{\circ} \mathrm{C}$ (Fig. 4c). The intensity of photoluminescence emission also started to decrease after annealing at $250^{\circ} \mathrm{C}$ (Fig. 4d). These techniques collectively demonstrate that $\mathrm{GeCH}_{3}$ begins to amorphize at $250^{\circ} \mathrm{C}$. Considering the lack of any PL in our previous studies on $\mathrm{GeH}$, the enhanced stability upon methyltermination is likely necessary to realize semiconductor properties such as band edge photoluminescence that are often disrupted by defect states.

\section{Discussion}

In summary, we have created for the first time $\mathrm{GeCH}_{3}$, a covalently modified direct band gap germanane, via a one-step topotactic metathesis reaction of $\mathrm{CaGe}_{2}$ crystals with $\mathrm{CH}_{3} \mathrm{I}$. We have shown that covalent methyl surface termination not only increases the band gap by $0.1 \mathrm{eV}$, but also enhances the thermal stability compared with $\mathrm{GeH}$. The photoluminescence quantum yield is on the same order of magnitude as other single-layer metal chalcogenides, but does not have the stringent single-layer requirement to observe such band edge emission, making these materials intriguing building blocks for future optoelectronic devices. This topotactic metathesis reaction can be extended to create new families of organic-terminated van der Waals materials from other solid-state crystal structures. Dimensionally reduced atomic-scale derivatives can have dramatically different properties than their parent analogue, which can be tuned with the nature of the surface substituent.

\section{Methods}

Synthesis. To synthesize $\mathrm{CaGe}_{2}$ crystals, $\mathrm{Ca}$ and $\mathrm{Ge}$ were loaded into a quartz tube with stoichiometric ratio. The quartz tube was sealed under vacuum and annealed at $950-1,050^{\circ} \mathrm{C}$ for $16-20 \mathrm{~h}$ and then slowly cooled down to room temperature ${ }^{9}$. To synthesize $\mathrm{GeCH}_{3}$, the $\mathrm{CaGe}_{2}$ crystals were loaded into an extraction thimble fully immersed in iodomethane (Sigma Aldrich), with a separated distilled water phase outside in the beaker and stir bar at the bottom of the extraction thimble (Supplementary Fig. 1). The reaction was running at room temperature for a week. Then the exfoliated flakes were rinsed with isopropanol (Sigma Aldrich), concentrated $\mathrm{HCl}(\mathrm{aq})$ (Fisher, Certified ACS Plus) followed by isopropanol. The sample was then dried on a Schlenk line at room temperature. For all thermal stability study experiments, the room temperature sample was annealed at different temperatures in flowing $5 \% \mathrm{H}_{2}$ in $\mathrm{Ar}$, then cooled down and characterized at room temperature.

Characterization. Powder XRD (Bruker D8 powder X-ray diffractometer, Rigaku MiniFlexII X-Ray diffractometer) and Single-crystal XRD (Nonius Kappa CCD diffractometer) were used to study the structure of $\mathrm{GeCH}_{3}$. FTIR measurements were collected on a Perkin-Elmer Frontier Dual-Range FIR/MidIR spectrometer that was loaded in an Ar-filled glovebox. XPS was collected using a Kratos Axis Ultra X-ray photoelectron spectrometer equipped with a monochromated (Al) $\mathrm{X}$-ray gun. The atomic force microscopy (AFM) images were collected on a Bruker AXS Dimension Icon Atomic/Magnetic Force Microscope with Scan Asyst. DRA measurements (Perkin-Elmer Lambda950 UV/Vis Spectrometer) and PL (Cary Eclipse Fluorescence Spectrophotometer) measurements were conducted to study the optical properties of the bulk solid crystals. In the PL measurements, the excitation wavelength was set to $380 \mathrm{~nm}$, the excitation and emission slit widths were set to $20 \mathrm{~nm}$ and $5 \mathrm{~nm}$, respectively. The absolute Quantum Yield of the solid samples was measured with the Quanta-phi (HORIBA Scientific) assembled in Fluorolog (HORIBA Scientific). The temperature-dependent and the thicknessdependent PL measurements were collected using a Renishaw InVia Raman equipped with a CCD detector upon excitation using a 633-nm HeNe laser at a power density of $\sim 24 \mathrm{~mW} \mathrm{~cm}^{-2}$, with a laser spot size of $\sim 2 \mu \mathrm{m}$ diameter. To collect the thickness-dependent measurement, we exfoliated $\mathrm{GeCH}_{3}$ onto $285 \mathrm{~nm} \mathrm{SiO}{ }_{2} / \mathrm{Si}$. The thicknesses of these flakes were measured by AFM to identify exfoliated flakes that had regions of relatively uniform thickness larger than the excitation spot size. The weighted average height from the AFM measurement was used to determine the thickness. For the temperature-dependent PL, exfoliated flakes were annealed at different temperatures in $5 \% \mathrm{H}_{2} / \mathrm{Ar}$, and their PL was recollected on the same flake after cooling down to room temperature. The same trend was observed for three different exfoliated flakes. Thermogravimetric Analysis (Q-500 thermogravimetric analyzer) was collected in flowing $\mathrm{N}_{2}$ at $10^{\circ} \mathrm{C} \mathrm{min}^{-1}$. Elemental Analysis (Atlantic Microlab Inc) of the $\mathrm{C} / \mathrm{H}$ ratio was collected to determine the ratio of $\mathrm{CH}_{3}$-termination to $\mathrm{H}$-termination.

Exfoliation of $\mathbf{G e C H}_{\mathbf{3}}$ flakes. To study the PL intensity of $\mathrm{GeCH}_{3}$ flakes with different thickness. The bulk $\mathrm{GeCH}_{3}$ flakes were exfoliated onto $285 \mathrm{~nm} \mathrm{SiO}_{2} / \mathrm{Si}$ substrate with kapton tape. The tape residue was cleaned with acetone and then by isopropanol, followed by a $\mathrm{N}_{2}$ blow dry to clean the residue solvent on the substrate. The thickness of these flakes was confirmed by AFM before the PL measurement. The AFM images are shown in Supplementary Fig. 4, the colour of the dots in AFM height profiles corresponds to the colour of the PL spectra in Fig. 3e.

Calculations. Density functional theory calculations were performed using the Vienna Ab initio simulation package ${ }^{37,38}$. The effect of the core electrons was included using projector augmented wave pseudopotentials ${ }^{39}$. To simulate $\mathrm{GeCH}_{3}$ monolayers, we used a supercell with a vacuum of $20 \AA$ and with the in-plane lattice parameter fixed to the experimental value $a=3.97 \AA$. Bilayer $\mathrm{GeCH}_{3}$ was simulated using a supercell with lattice parameters fixed to $a=3.97 \AA$ and $c=17.26 \AA$. Relaxation of the ionic positions was done using the Perdew-BurkeErnzerhof exchange correlation functional ${ }^{40,41}$ with a plane-wave cutoff energy of $600 \mathrm{eV}$ and a $9 \times 9 \times 1$ Monkhorst Pack k-point mesh ${ }^{42}$. To obtain an accurate description of the band gap, we performed high-level calculations based on the HSE06 hybrid functional ${ }^{31-33}$.

\section{References}

1. Novoselov, K. S. et al. Electric field effect in atomically thin carbon films. Science 306, 666-669 (2004).

2. Yang, X., Cheng, C., Wang, Y., Qiu, L. \& Li, D. Liquid-mediated dense integration of graphene materials for compact capacitive energy storage. Science 341, 534-537 (2013) 
3. Radisavljevic, B., Radenovic, A., Brivio, J., Giacometti, V. \& Kis, A. Single-layer $\mathrm{MoS}_{2}$ transistors. Nat. Nanotech. 6, 147-150 (2011).

4. Karunadasa, H. I. et al. A molecular $\mathrm{MoS}_{2}$ edge site mimic for catalytic hydrogen generation. Science 335, 698-702 (2012).

5. Mak, K. F., He, K., Shan, J. \& Heinz, T. F. Control of valley polarization in monolayer $\mathrm{MoS}_{2}$ by optical helicity. Nat. Nanotech. 7, 494-498 (2012).

6. Wang, Q. H., Kalantar-Zadeh, K., Kis, A., Coleman, J. N. \& Strano, M. S. Electronics and optoelectronics of two-dimensional transition metal dichalcogenides. Nat. Nanotech. 7, 699-712 (2012).

7. Chhowalla, M. et al. The chemistry of two-dimensional layered transition metal dichalcogenide nanosheets. Nat. Chem. 5, 263-275 (2013).

8. Wang, Q. H. et al. Understanding and controlling the substrate effect on graphene electron-transfer chemistry via reactivity imprint lithography. Nat. Chem. 4, 724-732 (2012).

9. Bianco, E. et al. Stability and exfoliation of germanane: a germanium graphane analogue. ACS Nano 7, 4414-4421 (2013).

10. Butler, S. Z. et al. Progress, challenges, and opportunities in two-dimensional materials beyond graphene. ACS Nano 7, 2898-2926 (2013).

11. Xu, Y. et al. Large-gap quantum spin Hall insulators in tin films. Phys. Rev. Lett. 111, 136804 (2013).

12. Elias, D. C. et al. Control of graphene's properties by reversible hydrogenation: evidence for graphane. Science 323, 610-613 (2009).

13. Englert, J. M. et al. Covalent bulk functionalization of graphene. Nat. Chem. 3, 279-286 (2011).

14. Cheng, S. H. et al. Reversible fluorination of graphene: evidence of a two-dimensional wide bandgap semiconductor. Phys. Rev. B 81, 205435 (2010).

15. Lebègue, S., Klintenberg, M., Eriksson, O. \& Katsnelson, M. I. Accurate electronic band gap of pure and functionalized graphane from $\mathrm{GW}$ calculations. Phys. Rev. B 79, 245117 (2009).

16. Mashtalir, O. et al. Intercalation and delamination of layered carbides and carbonitrides. Nat. Commun. 4, 1716 (2013).

17. Lukatskaya, M. R. et al. Cation intercalation and high volumetric capacitance of two-dimensional titanium carbide. Science 341, 1502-1505 (2013).

18. Roushan, M., Zhang, X. \& Li, J. Solution-processable white-light-emitting hybrid semiconductor bulk materials with high photoluminescence quantum efficiency. Angew. Chem. Int. Ed. 51, 436-439 (2012).

19. Dahn, J. R., Way, B. M., Fuller, E. \& Tse, J. S. Structure of siloxene and layered polysilane $\left(\mathrm{Si}_{6} \mathrm{H}_{6}\right)$. Phys. Rev. B 48, 17872-17877 (1993).

20. Okamoto, H. et al. Silicon nanosheets and their self-assembled regular stacking structure. J. Am. Chem. Soc. 132, 2710-2718 (2010).

21. Restrepo, O. D., Mishra, R., Goldberger, J. E. \& Windl, W. Tunable gaps and enhanced mobilities in strain-engineered silicane. J. Appl. Phys. 115, 033711 (2014).

22. Nakano, H. et al. Preparation of alkyl-modified silicon nanosheets by hydrosilylation of layered polysilane $\left(\mathrm{Si}_{6} \mathrm{H}_{6}\right)$. J. Am. Chem. Soc. 134, 5452-5455 (2012).

23. Van de Walle, C. G. \& Northrup, J. E. First-principles investigation of visible light emission from silicon-based materials. Phys. Rev. Lett. 70, 1116-1119 (1993).

24. Vogg, G., Brandt, M. S. \& Stutzmann, M. Polygermyne-a prototype system for layered germanium polymers. Adv. Mater. 12, 1278-1281 (2000).

25. Nemanick, E. J., Hurley, P. T., Brunschwig, B. S. \& Lewis, N. S. Chemical and electrical passivation of silicon (111) surfaces through functionalization with sterically hindered alkyl groups. J. Phys. Chem. B 110, 14800-14808 (2006).

26. Knapp, D., Brunschwig, B. S. \& Lewis, N. S. Chemical, electronic, and electrical properties of alkylated Ge(111) surfaces. J. Phys. Chem. C 114, 12300-12307 (2010).

27. Cardona, M. Vibrational spectra of hydrogen in silicon and germanium. Phys. Status Solidi B 118, 463-481 (1983).

28. Rivillon, S., Chabal, Y. J., Amy, F. \& Kahn, A. Hydrogen passivation of germanium (100) surface using wet chemical preparation. Appl. Phys. Lett. 87, 253101 (2005).
29. Griffiths, J. E. Infrared spectra of methylgermane, methyl- $\mathrm{d}_{3}$-germane, and methylgermane- $\mathrm{d}_{3}$. J. Chem. Phys. 38, 2879-2891 (1963).

30. Knapp, D., Brunschwig, B. S. \& Lewis, N. S. Transmission infrared spectra of $\mathrm{CH}_{3}-, \mathrm{CD}_{3}-$, and $\mathrm{C}_{10} \mathrm{H}_{21}-\mathrm{Ge}$ (111) surfaces. J. Phys. Chem. C 115, 16389-16397 (2011).

31. Heyd, J., Scuseria, G. E. \& Ernzerhof, M. Hybrid functionals based on a screened Coulomb potential. J. Chem. Phys. 118, 8207-8215 (2003).

32. Heyd, J., Scuseria, G. E. \& Ernzerhof, M. Erratum: 'Hybrid functionals based on a screened Coulomb potential' [J. Chem. Phys.118, 8207 (2003)]. J. Chem. Phys. 124, 219906 (2006).

33. Paier, J. et al. Screened hybrid density functionals applied to solids. J. Chem. Phys. 124, 154709 (2006).

34. Mak, K. F., Lee, C., Hone, J., Shan, J. \& Heinz, T. F. Atomically thin $\mathrm{MoS}_{2}$ : a new direct-gap semiconductor. Phys. Rev. Lett. 105, 136805 (2010).

35. Eda, G. et al. Photoluminescence from chemically exfoliated $\mathrm{MoS}_{2}$. Nano Lett 11, 5111-5116 (2011).

36. Splendiani, A. et al. Emerging photoluminescence in monolayer $\mathrm{MoS}_{2}$. Nano Lett. 10, 1271-1275 (2010).

37. Kresse, G. \& Hafner, J. Ab initio molecular-dynamics simulation of the liquid-metal-amorphous-semiconductor transition in germanium. Phys. Rev. B 49, 14251-14269 (1994).

38. Kresse, G. \& Hafner, J. Ab initio molecular dynamics for liquid metals. Phys. Rev. B 47, 558-561 (1993).

39. Blöchl, P. E. Projector augmented-wave method. Phys. Rev. B 50, 17953-17979 (1994).

40. Perdew, J. P., Burke, K. \& Ernzerhof, M. Generalized gradient approximation made simple. Phys. Rev. Lett. 77, 3865-3868 (1996).

41. Perdew, J. P., Burke, K. \& Ernzerhof, M. Generalized gradient approximation made simple [Phys. Rev. Lett. 77, 3865 (1996)]. Phys. Rev. Lett. 78, 1396-1396 (1997).

42. Monkhorst, H. J. \& Pack, J. D. Special points for Brillouin-zone integrations. Phys. Rev. B 13, 5188-5192 (1976).

\section{Acknowledgements}

We acknowledge the generosity of Professor Y. Wu for access to DRA absorption spectrometer. This work was supported in part by an allocation of computing time from the Ohio Supercomputing Center. We also acknowledge the Analytical Surface Facility at OSU chemistry, supported by National Science Foundation under grant number CHE-0639163. The synthesis, structure and characterization was supported by the Army Research Office (W911-NF-12-1-0481), and the theory simulations were supported by the Center for Emergent Materials at The Ohio State University, an NSF MRSEC at The Ohio State University (Grant DMR-0820414).

\section{Author contributions}

All experiments were designed by J.E.G. and S.J. All samples were synthesized and characterized by S.J. E.B. assisted with the synthesis and characterization of properties relative to $\mathrm{GeH}$. The XPS was performed by S.B. Density functional theory simulations were performed by O.D.R. and W.W. J.E.G. and S.J. wrote the manuscript with revisions from all the authors.

\section{Additional information}

Supplementary Information accompanies this paper at http://www.nature.com/ naturecommunications

Competing financial interests: The authors declare no competing financial interests.

Reprints and permission information is available online at http://npg.nature.com/ reprintsandpermissions/

How to cite this article: Jiang, S. et al. Improving the stability and optical properties of germanane via one-step covalent methyl-termination. Nat. Commun. 5:3389 doi: 10.1038/ncomms4389 (2014) 